\title{
The effect of listening to music on human transcriptome
}

Chakravarthi Kanduri, Pirre P Raijas, Minna Ahvenainen, Anju K Philips, Liisa Ukkola-Vuoti, Harri Lähdesmäki, Irma E Järvelä

Although brain imaging studies have demonstrated that listening to music alters human brain structure and function, the molecular mechanisms mediating those effects remain unknown. With the advent of genomics and bioinformatics approaches, these effects of music can now be studied in a more detailed fashion. To verify whether listening to classical music has any effect on human transcriptome, we performed genome-wide transcriptional profiling from the peripheral blood of participants after listening to classical music $(n=48)$, and after a control study without music exposure $(n=15)$. As musical experience is known to influence the responses to music, we compared the transcriptional responses of musically experienced and inexperienced participants separately with those of the controls. Comparisons were made based on two subphenotypes of musical experience: musical aptitude and music education. In musically experiencd participants, we observed the differential expression of 45 genes ( 27 up- and 18 down-regulated) and 97 genes (75 up- and 22 down-regulated) respectively based on subphenotype comparisons (rank product non-parametric statistics, pfp 0.05, > 1.2-fold change over time across conditions). Gene ontological overrepresentation analysis (hypergeometric test, FDR $<0.05$ ) revealed that the up-regulated genes are primarily known to be involved in the secretion and transport of dopamine, neuron projection, protein sumoylation, long-term potentiation and dephosphorylation. Down-regulated genes are known to be involved in ATP synthase-coupled proton transport, cytolysis, and positive regulation of caspase, peptidase and endopeptidase activities. One of the most up-regulated genes, alphasynuclein (SNCA), is located in the best linkage region of musical aptitude on chromosome $4 q 22.1$ and is regulated by GATA2, which is known to be associated with musical aptitude. Several genes reported to regulate song perception and production in songbirds displayed altered activities, suggesting a possible evolutionary conservation of sound perception between species. We observed no significant findings in musically inexperienced participants. 


\section{The effect of listening to music on human transcriptome}

3 Chakravarthi Kanduri ${ }^{1}$, Pirre Raijas ${ }^{2}$, Minna Ahvenainen ${ }^{1}$, Anju K Philips ${ }^{1}$, Liisa Ukkola-Vuoti ${ }^{1}$, Harri

4 Lähdesmäki ${ }^{3}$, Irma Järvelä ${ }^{*}$

5

6 'Department of Medical Genetics, University of Helsinki, P.O. Box 720, 00014 University of Helsinki,

7 Finland

8 2DocMus Department, University of the Arts Helsinki, P.O. Box 86, 00251 Helsinki, Finland

$9{ }^{3}$ Department of Information and Computer Science, Aalto University, FI-00076 AALTO, Finland

10

$11 *$ Corresponding author

12 Irma Järvelä, $\mathrm{MD} \mathrm{PhD}$

13 Department of Medical Genetics

14 University of Helsinki

15 Box 720, 00251 Helsinki, FINLAND

16 Tel: +358 505447030

17 E-mail: irma.jarvela@helsinki.fi (IJ)

18

19

20

21 


\section{Introduction}

25 Listening to music is common in all societies. A plethora of neurophysiological studies have

26 demonstrated that listening to and/or playing music has multiple measurable effects on human brain

27 structure and function (Elbert et al., 1995; Blood \& Zatorre, 2001; Sutoo \& Akiyama, 2004; Koelsch,

28 2011; Salimpoor et al., 2011, 2013; Herholz \& Zatorre, 2012; Chanda \& Levitin, 2013). In

29 investigations using positron emission tomography (PET), music listening has been reported to cause

30 physiological changes in cerebral blood flow, cardiovascular and muscle function, and enhanced

31 dopamine secretion in the human brain (Sutoo \& Akiyama, 2004; Angelucci et al., 2007; Salimpoor et

32 al., 2011). Music has been demonstrated to regulate emotions and evoke pleasure, primarily through its

33 action on the brain's reward centres like the limbic and mesolimbic structures including the nucleus

34 accumbens, hypothalamus, subcallosal cingulate gyrus, pre-frontal anterior cingulate, and hippocampus

35 (Brown, Martinez \& Parsons, 2004; Koelsch, 2011, 2014; Salimpoor et al., 2011, 2013). Music has

36 also been used as a therapeutic tool in clinical settings. (AMTA; Conrad, 2010; Holmes, 2012).

37 However, the molecular mechanisms and biological pathways mediating the effects of music remain

38 unknown.

39 Genomics and bioinformatics offer methods (Lander, 2011) to explore the biology and evolution of

40 music and sounds at the molecular level. To date, few genome-wide scans have been performed in

41 musical traits in humans (Asher et al., 2008; Pulli et al., 2008; Park et al., 2012; Oikkonen et al., 2014).

42 Genome-wide expression analysis can be applied to study human traits in an unbiased, hypothesis-free

43 fashion based on their molecular properties, rather than anatomic regions. Here, we have utilized a

44 combination of genomic and bioinformatic methods to analyze the effect of classical music on the 
45 peripheral whole blood transcriptome. Peripheral blood was used, as brain samples are inaccessible in 46 humans.

\section{Materials and Methods}

\section{Ethics statement}

50 The Ethics Committee of Helsinki University Central Hospital approved this study. Written informed 51 consent was obtained from all the participants.

52 53

54 A total of 48 individuals (aged 18-73; mean 42.5) participated in the study (Table S1). All the 48 55 56

\section{Participants and phenotypes} participants were characterized for musical aptitude and music education. Musical aptitude was measured using three tests: the auditory structuring ability test (Karma music test) (Karma, 2007), and the Seashore tests for pitch and for time (Seashore, Lewis \& Saetveit, 1960). Details of the tests have been described in Oikkonen et al (2014) (Oikkonen et al., 2014). A combined score of the three tests (COMB score; range 0-150) was used to define musical aptitude. COMB scores were classified as either high or low based on the upper and lower quartiles of score distribution. Data on music education were collected using a questionnaire. The self-reported levels of music education (referred to as edu classes 1-4), received through studies or degrees from music schools, institutes, or universities, were classified as follows: 1 represents no music education; 2 represents music education of less than two years; 3 represents music education of more than ten years; and 4 represents a professional musician. The approximate time of systematic music education and training was 21.42 years on average, in participants of edu classes 3-4. Table 1 shows the sample's phenotype characteristics. 


\section{Exposure to music}

69 To our knowledge, no previous studies have systematically studied the effect of listening to music on

70 genome-wide transcriptional profiles of humans. We have previously shown that music-listening habits

71 vary a lot among listeners (Ukkola-Vuoti et al., 2011). To start with, we chose to study the effect of

72 classical music, Wolfgang Amadeus Mozart's Violin Concerto No. 3 in G major, K.216 because it is

73 relatively familiar in the western culture. As the human brain perceives complex sounds in a

74 millisecond-level time frame (Wang et al., 2009; Kayser, Logothetis \& Panzeri, 2010), we expected

75 that the 20 minute-listening session (duration that the concerto lasts) will induce an effect of music on

76 human transcriptome. In studies on the effect of pain in humans, very short durations of pain induction

77 (8 to 150 seconds) have been used (Hubbard et al., 2011). The participants were unaware of the type of

78 music that was intended for the listening session. Peripheral blood samples were collected from all the

7948 participants just before and after the listening session. From here on, participants who listened to 80 music are referred to as listeners throughout the text.

\section{Control study}

82

83 The same 48 participants were invited to a control study. Of these, 15 participants could attend. The

84 participants were advised to avoid listening to music and hard exercise the day before the control study.

85 The control study was performed in a 'music-free' environment, where the participants had an

86 opportunity to converse, read a magazine, or take a walk outside (no exercise) during the session.

87 Peripheral blood samples were collected from the participants just before and after 20 minutes in the 88 control session (the same duration as in the listening session). From here on, participants of the control 89 session are referred to as controls throughout the text. 


\section{Genome-wide expression profilling}

92 For this procedure, 2 X $2.5 \mathrm{ml}$ samples of peripheral blood were drawn into PAXgene blood RNA tubes

93 (PreAnalytiX GmbH, Hombrechtikon, Switzerland) as per the kit instructions, in both of the sessions.

94 Total RNA was isolated using the PAXgene blood miRNA Kit (PreAnalytiX GmbH, Hombrechtikon,

95 Switzerland) as per the kit manual. Purified RNA samples were measured for concentration and purity

96 on the NanoDrop 1000 v.3.7 (Thermo Fisher Scientific, USA). Globin mRNA was depleted from our

97 samples using Ambion's Human GLOBINclear ${ }^{\mathrm{TM}}$ kit (Applied Biosystems, USA) as per the kit insert.

98 The samples were measured on the NanoDrop 1000 to determine the sample concentration and purity

99 and for integrity on the 2100 Bioanalyzer (Agilent Technologies, Germany) before being diluted to

$10050 \mathrm{ng} / \mu \mathrm{l}$ using RNase-free water. A total of $2 \mu \mathrm{g}$ of RNA was assayed on the Illumina HumanHT-12 v4

101 bead array (Illumina Inc.; San Diego, CA, USA), which targets more than 47,000 probes. The gene

102 expression profiling assays for the listening and control sessions were conducted in two separate

103 batches. To account for the batch effect corrections, six samples from the listening session were

104 assayed together with the control session samples. Intensity data were exported through Bead Studio

105 software. The data reported in this article have been deposited in the Gene Expression Omnibus

106 database, www.ncbi.nlm.nih.gov/geo (accession no. GSE15180).

\section{Data preprocessing}

108

109 The Lumi package was used to read and preprocess the signal intensity data. Specifically, pre-

110 processing included background correction, variance stabilizing transformation, and quantile

111 normalization. Data from both the listening $(n=48)$ and control $(n=15)$ sessions were normalized

112 separately. Five samples from the control session were excluded from further analyses owing to data

113 quality. In addition, we used the ComBat method (Johnson, Li \& Rabinovic, 2007) to adjust for batch 
114 effects and determined if this correction affected the pre-post fold-changes across conditions. However,

115 we did not find significant differences between the fold-changes of corrected and uncorrected data over

116 time across conditions. Therefore, we chose to proceed with the uncorrected data owing to the

117 strengths of our analysis methods as described below. After normalization, duplicate and un-annotated

118 probes were excluded using the genefilter package (R package version 1.40.0). Before extracting the

119 expression values from the normalized data, Illumina's detection p-values (threshold: 0.01 ) were used

120 to filter out probes with low intensities corresponding to the background signal. Finally, only those

121 probes that were expressed in at least half of all of the arrays (listening and control sessions) were 122 chosen for the study.

\section{Differential expression analysis}

125 The choice of an appropriate statistical test for the identification of differentially expressed genes

126 depends upon several aspects of the data including the underlying distribution, homogeneity, and the 127 sample size. As the statistical tests for normality are known to be sensitive to sample size, we used a 128 normal Q-Q plot to get a glimpse of the distribution of the data. For this, we randomly visualized the 129 distribution of transcriptional responses of control samples $(n=10)$ for several transcripts (Fig. S1) 130 using normal Q-Q plots. We observed that the data appeared to deviate from normality in several 131 instances. As the central limit theorem does not always hold true for small sample sizes, a cautious 132 approach here would be to employ a non-parametric test (better being safe than sorry). Non-parametric 133 tests are deemed to be appropriate analysis tools when the distribution of data is difficult to 134 characterize, because they make less stringent distributional assumptions. Therefore, we chose to use 135 the rank product (Breitling et al., 2004) non-parametric test statistic, which is relatively powerful 136 especially for small sample sizes, and when the data is heterogeneous and does not meet normality.

137 Rank product-based methods outperformed several other methods including empirical Bayes statistic 
138 (limma) and SAM, when the sample size is small and when the data is non-homogeneous (Jeffery,

139 Higgins \& Culhane, 2006). Also, a comparison of eight gene ranking methods using Microarray

140 Quality Control datasets (golden-standard) has demonstrated the high sensitivity and specificity of the 141 rank product method (Kadota \& Shimizu, 2011).

142 To identify the differentially expressed genes, we compared the magnitude of pre-post changes in gene 143 expression across conditions using the rank product method implemented in the RankProd 144 Bioconductor package. Based on the estimated percentage of false predictions (pfp), RankProd 145 employs a non-parametric statistic to identify genes that are consistently ranked high among the most 146 up- or down-regulated genes in replicate experiments. Instead of analyzing the actual expression value, 147 this method utilizes the ranks of genes in each sample. The strength of rank product method allows us 148 to compare and combine the datasets of the listening and control studies. After the identification of 149 differentially expressed genes using a pfp of 0.05 in RankProd, we selected only those genes that 150 exceeded an effect-size cut-off ( $>1.2$-fold change over time across conditions, and at least a pre-post 151 change of $10 \%$ in gene expression in the listening session). Here, we would like to point out a couple 152 of aspects of these selection criteria. First, pfp employed by RankProd is equivalent to the standard 153 false discovery rate (FDR). Second, there exists a widespread misconception that only two-fold 154 changes are significant (Hoheisel, 2006) and that false notion is based on the very initial publications 155 of microarray studies, which used a two-fold change criteria for a particular group of experiments 156 because of biological relevance. Fold-change thresholds are completely arbitrary and in the majority of 157 the cases they depend upon the underlying biological question. For example, studies that investigated 158 the effect of gene-environment interactions (socio-environmental effect (Cole et al., 2007), yogic 159 meditation effect (Black et al., 2012)) used unorthodox fold-change thresholds.

160 We further performed successive functional annotation analyses using GeneTrail 
161 (http://genetrail.bioinf.uni-sb.de/) and IPA (Ingenuity ${ }^{\circledR}$ Systems, www.ingenuity.com). The gene

162 ontological overrepresentation analysis in GeneTrail uses a hypergeometric distribution test along with

163 a conservative multiple testing correction method $(\mathrm{FDR}<0.05)$ to assess whether genes belonging to

164 certain functional categories are enriched in the dataset. In addition to the standard gene ontology

165 analyses, we performed upstream transcription regulator analysis, which essentially predicts all the

166 upstream transcription regulators (transcription factors, receptors, cytokines, microRNA, and kinases)

167 that could have possibly mediated the observed differential expression. Based on the overlap between

168 known targets of a transcription regulator and the set of differentially expressed genes, an overlap p-

169 value is computed using Fisher's exact test $(\mathrm{p}<0.01)$. Further, the activation states of the predicted

170 transcription regulators are also inferred using an activation Z-score, which is based on literature-

171 derived knowledge on the direction of regulation (either activating or inhibiting).

\section{Results}

173

174 175

176

177

178

179

180

181 reference for the comparative analyses.

182 We used Spearman's rank-based correlation to check for each gene, whether the transcriptional 183 responses correlated with the age or sex of the participants in either the listening session $(n=48)$ or the 
184 control sessions $(\mathrm{n}=10)$. After multiple testing corrections, we found no significant effects of age or sex 185 on the transcriptional responses.

186 187

188 189

190 191

194

195

\section{Transcriptional response after listening to music}

Based on neuroscientific studies, the brains of musicians and non-musicians differ structurally and functionally (Elbert et al., 1995; Gaser \& Schlaug, 2003). This led us to ask a question, whether the transcriptional responses of musically experienced participants would differ from those of musically inexperienced participants when listening to music. Therefore, we compared the transcriptional responses of listening to music separately for musically experienced and inexperienced participants vs controls. Comparisons were made based on two subphenotypes of musical experience: musical aptitude and music education.

First, we compared the magnitude of pre-post fold-changes in the genome-wide transcriptional profiles of listeners of edu classes 3-4 $(\mathrm{n}=29)$ and controls $(\mathrm{n}=10)$. Using RankProd non-parametric statistics and stringent selection criteria, we identified 45 differentially expressed genes (27 up-regulated and 18 down-regulated). Next, we compared the genome-wide transcriptional profiles of listeners with high COMB scores $(\mathrm{n}=12)$ and controls $(\mathrm{n}=10)$. Similar statistical analysis identified 97 differentially expressed genes (75 up-regulated and 22 down-regulated). The differentially expressed genes from both the comparisons are listed in Table S2, and a comparison of the pre-post changes in both conditions is shown in Fig. 1.

\section{Functional annotation}

205 Based on gene ontology analyses (Table S3), the genes up-regulated in the listeners of edu classes 3-4 are known to be primarily involved in the regulation, secretion and transport of the neurotransmitter 
207 dopamine (e.g., SNCA, RTN4, and SLC6A8), protein sumoylation (SUMO2 and HDAC4) and neuron 208 projection (SNCA, RTN4, DICER1 and MYC). Down-regulated genes are known to affect functions 209 such as mitochondrial ATP synthase-coupled proton transport and cytolysis (e.g., ATP5J, ATP5L, $210 G Z M H$, and GZMA). Several of the genes, including the dopamine secretion-related genes (SNCA,

211 RTN4) up-regulated in listeners of edu classes 3-4, were also found to be up-regulated in listeners with 212 high COMB scores. Here, we should note that the COMB scores are strongly correlated with music edu 213 classes (Spearman's rho 0.5644; p-value 2.931e-05). In listeners with high COMB scores, gene 214 ontology classification revealed that the up-regulated genes are involved in functions such as long-term 215 synaptic potentiation ( $N P T N$ and $S N C A$ ), dephosphorylation and regulation of cell communication.

216 Down-regulated genes are known to be involved in functions such as positive regulation of caspase, 217 peptidase and endopeptidase activities (Table S3).

218 We further performed Entrez gene annotation and an extensive literature survey for all the 219 genes that are differentially expressed after listening to music (in listeners of both edu classes 3-4 and 220 high COMB scores). This revealed that the up-regulated genes are known to be associated with 221 dopamine signaling, synaptic neurotransmission, synaptic function, learning, memory and cognitive 222 performance, song learning and singing in songbirds, auditory cortical activation, absolute pitch, 223 neuroprotection and neurogenesis (Tables 2, S4). On the other hand, down-regulated genes are known 224 to cause mammalian neuronal apoptosis, immoderate oxidative phoshorylation and deficits in 225 dopaminergic neurotransmission, which are the characteristics of neurodegeneration (Tables 2, S4). 226 Detailed information about the putative biological functions of the differentially expressed genes is 227 provided in Table $\mathbf{S 4}$.

Upstream regulator analysis

230 To obtain insight into the molecules that might mediate the observed differences in gene expression, 
231 we performed an upstream transcription regulator analysis using IPA (Table S5). This analysis

232 revealed that the up-regulated genes in listeners of edu classes 3-4 significantly overlap the known

233 target genes of the glucocorticoid receptor $N R 3 C 1$ ( $\mathrm{p}$-value 0.001 ), and progesterone receptor $P G R$ ( $\mathrm{p}$ -

234 value 0.0008 ), whereas the down-regulated genes did not display any significant overlap. In listeners

235 with high COMB scores, upstream regulator analysis again identified the up-regulation of target genes

236 of the glucocorticoid receptor gene $(\mathrm{NR} 3 \mathrm{Cl})$ and also the target genes of several other transcription

237 regulators such as TP53, MYC, HOXA9, CD24 and chorionic gonadotropins. Down-regulated genes

238 significantly overlapped the known target genes of pro-inflammatory transcription regulators such as

239 tumor necrosis factor $(T N F)$, a member of its superfamily (TNFSF10), interferon gamma (IFNG), a

240 member of its gene cluster (IFNA2), the microtubule-associated protein tau (MAPT) and the nuclear

241 factor kappa B family gene (NFKB1A; Table S5).

242 Transcriptional responses of participants with no significant experience

243

244 Furthermore, we repeated similar analyses to compare the magnitude of pre-post fold-changes over

245 time in listeners of edu classes 1-2 vs controls and listeners with low COMB scores vs controls. Using

246 the same analysis methods and selection criteria to identify the differentially expressed genes, we

247 identified 8 and 22 differentially expressed genes, respectively in the comparisons. However,

248 functional characterization of those genes did not reveal any significant findings.

\section{Discussion}

The findings of this study suggest that listening to classical music has an effect on human

252 transcriptome. The up-regulation of genes related to dopamine secretion and signaling is in agreement

253 with the previous neuroimaging-based evidences (Salimpoor et al., 2011). Particularly, alpha- 
synuclein ( $\alpha$-synuclein; $S N C A)$, one of the most up-regulated genes, is involved in dopamine (DA)

255

256 neuronal homeostasis (Murphy et al., 2000; Oczkowska et al., 2013). Interestingly, SNCA is located on chromosome 4q22.1, the most significant region of linkage for musical aptitude (Pulli et al., 2008; Oikkonen et al., 2014) and regulated by GATA2 (Scherzer et al., 2008), which is associated with musical aptitude (Oikkonen et al., 2014) (Fig. 2). These data provide convergent evidence about the molecular basis of musical traits from both DNA and RNA studies. Another finding from the upstream regulator analysis suggests that listening to music primarily increased the expression of the target genes of the glucocorticoid receptor $(\mathrm{NR} 3 \mathrm{Cl})$. Notably, dopaminoceptive neuronal glucocorticoid receptor has been described as a key molecule in the regulation of addictive behavior. By reducing dopamine reuptake, NR3C1 increases the synaptic concentration of dopamine, which leads to rewarding and reinforcing properties (Ambroggi et al., 2009) that have previously been linked to listening to music (Blood \& Zatorre, 2001; Koelsch, 2011, 2014; Salimpoor et al., 2011, 2013).

The up-regulation of genes related to synaptic vesicular exocytosis, endocytosis, neurotransmission and plasticity seems perfectly rational here because, the majority of these biological processes are essential for the secretion and signaling of neurotransmitters (Südhof \& Rizo, 2011; Saheki \& De Camilli, 2012). As listening to music has been known to induce the secretion and signaling of a neurotransmitter, dopamine (Sutoo \& Akiyama, 2004; Salimpoor et al., 2011), we can speculate the role of these up-regulated genes in facilitating dopaminergic neurotransmission after listening to music. Moreover, some of the up-regulated genes have evident roles in enhancing cognitive functions like long-term potentiation (LTP) and memory. In previous behavioral studies, music education and training have proven to have beneficial effects on cognitive development, cognitive performance, verbal and long-term memories (Rammsayer \& Brandler, 2003; Schlaug et al., 2005; Sluming et al., 2007; Wong et al., 2007; Roden, Kreutz \& Bongard, 2012; Rodrigues, Loureiro \& Caramelli, 2013). 
Several of the differentially expressed genes have been demonstrated to be responsible for song

279 learning and singing in songbirds (Wada et al., 2006), which suggests a possible evolutionary 280 conservation in biological processes related to sound perception. Furthermore, the up-regulation of 281 genes associated with human auditory cortical activation (Renvall et al., 2012) and absolute pitch 282 (Theusch, Basu \& Gitschier, 2009; Gervain et al., 2013) are logical, because listening to music 283 involves both of those auditory phenomena.

Auditory perception processes have been known to exhibit convergent evolution across species.

285 Notably, the human auditory center is identical to those of the first primates who inhabited the planet 286 millions of years ago (Langner et al., 2003; Montealegre-Z et al., 2012). In addition, widespread 287 adaptive convergent sequence evolution has been found recently in hearing-related genes in 288 echolocating bats and dolphins (Parker et al., 2013). Similarly, convergent sequence evolution has also 289 been identified in vocal-learning birds and mammals (Zhang et al., 2014). More recently, convergent 290 gene expression specializations have been detected in songbirds and humans in the regions of brain that 291 are essential for auditory perception and speech production (Pfenning et al., 2014). Thus, the genes 292 detected by Pfenning et al, in general, represent the genes belonging to auditory perception pathway in 293 both songbirds and humans. Here, genes belonging to the auditory perception pathway $(\sim 2$-fold 294 enrichment; p-value: 0.028; two-sided Fisher's exact test) were found to be enriched among the genes 295 that are differentially expressed after listening to music. This suggests that our results serve as a 296 relevant molecular background for music perception in humans. The widely-documented neuroprotective role of some of the up-regulated genes and the downregulation of several neurodegeneration-inducing genes support the notion of a neuroprotective role for music and may provide a working mechanism for the use of music therapy, especially in treating 300 neurodegenerative diseases (AMTA; Conrad, 2010; Holmes, 2012). 
In this study, significant transcriptional responses were observed only in individuals who either

302 have substantial periods of music education/training or have relatively higher musical aptitude scores.

303 This suggests that certain musical abilities (either innate or acquired through music education) may

304 influence the transcriptional responses of listening to music. Previous works have shown that the

305 familiarity of music attained through music education or repeated music exposure is known to largely

306 influence the rewarding aspects of listening to music (Sarkamo et al., 2008; Salimpoor et al., 2009; van

307 den Bosch, Salimpoor \& Zatorre, 2013; Schubert, Hargreaves \& North, 2014). Here, we acknowledge

308 that the effect of music exposure on human gene expression could be very subjective and may vary

309 depending on several factors such as age, sex, culture, previous listening habits, music education and

310 training and personal liking of music, as recently discussed (Wong et al., 2007; Salimpoor et al., 2009;

311 van den Bosch, Salimpoor \& Zatorre, 2013; Mikutta et al., 2014). To be able to comprehensively

312 characterize the transcriptomic alterations of music-listening, further studies are required to assess the

313 effect of listening to different genres of music, at different ages, in different ethnicities, in individuals

314 with varying music education levels and listening habits, with varying durations of listening.

315 As brain samples are inacesssibile in this type of study, we used peripheral blood as a window to the

316 study the effects of listening to music. Peripheral blood is known to share more than $80 \%$ of the

317 transciptome and significant gene expression similarities with other tissues including multiple regions

318 of brain (Liew et al., 2006; Sullivan, Fan \& Perou, 2006; Tylee, Kawaguchi \& Glatt, 2013). Thus

319 peripheral blood could certainly provide surrogate information concerning gene expression in brain

320 tissue for a subset of genes (Davies et al., 2009). For instance, the molecular alterations in dopamine

321 metabolism and mitochondrial function, which are the potential hallmarks of Parkinson's disease, have

322 been detected in peripheral blood (Scherzer et al., 2007). Notably, genes that are responsive to 323 physiological stimuli (which are earlier thought to be tissue-specific) and genes involved in 
neuroendocrine pathways (e.g, hormone receptors, neurotransmitter receptors) are expressed in the peripheral blood. Because of these characteristics, peripheral blood has been used as a proxy in several studies when a specific tissue is not available (e.g, human brain), especially in behavioral and neurodegenerative studies (Mohr \& Liew, 2007). In the wake of all these findings, a subset of the molecular mechanisms identified here may legitimately reflect the transcriptomic alterations in brain after listening to music.

\section{Acknowledgements}

We thank all of the participants for their generous cooperation. We thank Päivi Onkamo for her constructive comments about the manuscript. We are grateful to Petri Myllynen, Sanna Pyy, Laura Salmela, Sonja Suhonen, Jaana Oikkonen and Kai Karma for expert technical help. We thank the HighThroughput Genomics Group at the Wellcome Trust Centre for Human Genetics for the generation of the Gene Expression data.

\section{References}

Ambroggi F, Turiault M, Milet A, Deroche-Gamonet V, Parnaudeau S, Balado E, Barik J, van der Veen R, Maroteaux G, Lemberger T et al. 2009. Stress and addiction: glucocorticoid receptor in dopaminoceptive neurons facilitates cocaine seeking. Nature neuroscience 12:247-249.

AMTA 2014. Music Therapy Fact Sheets \& Bibliographies, American Music Therapy Association (AMTA).

Angelucci F, Ricci E, Padua L, Sabino A, Tonali PA. 2007. Music exposure differentially alters the levels of brain-derived neurotrophic factor and nerve growth factor in the mouse hypothalamus. Neurosci Lett 429:152-155.

Asher JE, Lamb JA, Brocklebank D, Cazier JB, Maestrini E, Addis L, Sen M, Baron-Cohen S, Monaco AP. 2008. A whole-genome scan and fine-mapping linkage study of auditory-visual synesthesia reveals rvidence of linkage to chromosomes 2q24, 5q33, 6p12, and 12p12. American Journal of Human Genetics 84:279-285. 
Black DS, Cole SW, Irwin MR, Breen E, St Cyr NM, Nazarian N, Khalsa DS, Lavretsky H. 2012. Yogic meditation reverses NF- $\mathrm{B}$ and IRF-related transcriptome dynamics in leukocytes of family dementia caregivers in a randomized controlled trial. Psychoneuroendocrinology.

Blood AJ, Zatorre RJ. 2001. Intensely pleasurable responses to music correlate with activity in brain regions implicated in reward and emotion. Proc Natl Acad Sci U S A 98:11818-11823.

Van den Bosch I, Salimpoor VN, Zatorre RJ. 2013. Familiarity mediates the relationship between emotional arousal and pleasure during music listening. Frontiers in human neuroscience 7:534.

Breitling R, Armengaud P, Amtmann A, Herzyk P. 2004. Rank products: A simple, yet powerful, new method to detect differentially regulated genes in replicated microarray experiments. FEBS Letters 573:83-92.

Brown S, Martinez MJ, Parsons LM. 2004. Passive music listening spontaneously engages limbic and paralimbic systems. NeuroReport 15:2033-2037.

Chanda ML, Levitin DJ. 2013. The neurochemistry of music. Trends Cogn Sci 17:179-193.

Cole SW, Hawkley LC, Arevalo JM, Sung CY, Rose RM, Cacioppo JT. 2007. Social regulation of gene expression in human leukocytes. Genome Biol 8:R189.

Conrad C. 2010. Music for healing: from magic to medicine. Lance 376:1980-1981.

Davies MN, Lawn S, Whatley S, Fernandes C, Williams RW, Schalkwyk LC. 2009. To what extent is blood a reasonable surrogate for brain in gene expression studies: Estimation from mouse hippocampus and spleen. Frontiers in Neuroscience 1.

Elbert T, Pantev C, Wienbruch C, Rockstroh B, Taub E. 1995. Increased cortical representation of the fingers of the left hand in string players. Science (New York, N.Y.) 270:305-307.

Gaser C, Schlaug G. 2003. Brain structures differ between musicians and non-musicians. The Journal of neuroscience : the official journal of the Society for Neuroscience 23:9240-9245.

Gervain J, Vines BW, Chen LM, Seo RJ, Hensch TK, Werker JF, Young AH. 2013. Valproate reopens critical-period learning of absolute pitch. Frontiers in systems neuroscience 7:102.

Herholz SC, Zatorre RJ. 2012. Musical training as a framework for brain plasticity: behavior, function, and structure. Neuron 76:486-502.

Hoheisel JD. 2006. Microarray technology: beyond transcript profiling and genotype analysis. Nature reviews. Genetics 7:200-210.

Holmes D. 2012. Music therapy’s breakthrough act. Lancet neurology 11:486-7. 
Hubbard CS, Ornitz E, Gaspar JX, Smith S, Amin J, Labus JS, Kilpatrick LA, Rhudy JL, Mayer EA, Naliboff BD. 2011. Modulation of nociceptive and acoustic startle responses to an unpredictable threat in men and women. Pain 152:1632-1640.

Jeffery IB, Higgins DG, Culhane AC. 2006. Comparison and evaluation of methods for generating differentially expressed gene lists from microarray data. BMC bioinformatics 7:359.

Johnson WE, Li C, Rabinovic A. 2007. Adjusting batch effects in microarray expression data using empirical Bayes methods. Biostatistics (Oxford, England) 8:118-127.

Kadota K, Shimizu K. 2011. Evaluating methods for ranking differentially expressed genes applied to microArray quality control data. BMC bioinformatics 12:227.

Karma K. 2007. Musical aptitude definition and measure validation: Ecological validity can endanger the construct validity of musical aptitude tests. Psychomusicology: A Journal of Research in Music Cognition 19:79-90.

Kayser C, Logothetis NK, Panzeri S. 2010. Millisecond encoding precision of auditory cortex neurons. Proceedings of the National Academy of Sciences of the United States of America 107:1697616981.

Koelsch S. 2011. Toward a neural basis of music perception - a review and updated model. Front Psychol 2:110.

Koelsch S. 2014. Brain correlates of music-evoked emotions. Nature Reviews Neuroscience 15:170180.

Lander ES. 2011. Initial impact of the sequencing of the human genome. Nature 470:187-197.

Langner G, Simonis C, Braun S, Ochse M. 2003. Evidence for a Pitch Helix in the Ventral Nucleus of the Lateral Lemniscus in the Gerbil. In: Association for Research in ... 1996.

Liew CC, Ma J, Tang HC, Zheng R, Dempsey AA. 2006. The peripheral blood transcriptome dynamically reflects system wide biology: a potential diagnostic tool. J Lab Clin Med 147:126132.

Mikutta C, Maissen G, Altorfer A, Strik W, Koenig T. 2014. Professional musicians listen differently to music. Neuroscience 268C:102-111.

Mohr S, Liew CC. 2007. The peripheral-blood transcriptome: new insights into disease and risk assessment. Trends in Molecular Medicine 13:422-432.

Montealegre-Z F, Jonsson T, Robson-Brown KA, Postles M, Robert D. 2012. Convergent evolution between insect and mammalian audition. Science (New York, N.Y.) 338:968-71. 
411 Murphy DD, Rueter SM, Trojanowski JQ, Lee VM. 2000. Synucleins are developmentally expressed, and alpha-synuclein regulates the size of the presynaptic vesicular pool in primary hippocampal neurons. The Journal of neuroscience : the official journal of the Society for Neuroscience 20:3214-20.

Oczkowska A, Kozubski W, Lianeri M, Dorszewska J. 2013. Mutations in PRKN and SNCA Genes Important for the Progress of Parkinson's Disease. Current genomics 14:502-17.

Oikkonen J, Huang Y, Onkamo P, Ukkola-Vuoti L, Raijas P, Karma K, Vieland VJ, Järvelä I. 2014. A genome-wide linkage and association study of musical aptitude identifies loci containing genes related to inner ear development and neurocognitive functions. Molecular psychiatry:1-8.

Park H, Lee S, Kim H-J, Ju YS, Shin J-Y, Hong D, von Grotthuss M, Lee D-S, Park C, Kim JH et al. 2012. Comprehensive genomic analyses associate UGT8 variants with musical ability in a Mongolian population. Journal of Medical Genetics.

Parker J, Tsagkogeorga G, Cotton J a, Liu Y, Provero P, Stupka E, Rossiter SJ. 2013. Genome-wide signatures of convergent evolution in echolocating mammals. Nature 502:228-31.

Pfenning AR, Hara E, Whitney O, Rivas M V., Wang R, Roulhac PL, Howard JT, Wirthlin M, Lovell P V., Ganapathy G et al. 2014. Convergent transcriptional specializations in the brains of humans and song-learning birds. Science 346:1256846-1256846.

Pulli K, Karma K, Norio R, Sistonen P, Goring HH, Jarvela I. 2008. Genome-wide linkage scan for loci of musical aptitude in Finnish families: evidence for a major locus at 4q22. J Med Genet $45: 451-456$.

Rammsayer TH, Brandler S. 2003. Differences in Mental Abilities between Musicians and NonMusicians. Psychology of Music 31:123-138.

Renvall H, Salmela E, Vihla M, Illman M, Leinonen E, Kere J, Salmelin R. 2012. Genome-wide linkage analysis of human auditory cortical activation suggests distinct loci on chromosomes 2, 3, and 8. The Journal of neuroscience : the official journal of the Society for Neuroscience 32:14511-8.

Roden I, Kreutz G, Bongard S. 2012. Effects of a school-based instrumental music program on verbal and visual memory in primary school children: a longitudinal study. Frontiers in psychology $3: 572$.

Rodrigues AC, Loureiro MA, Caramelli P. 2013. Long-term musical training may improve different forms of visual attention ability. Brain and cognition 82:229-35.

Saheki Y, De Camilli P. 2012. Synaptic vesicle endocytosis. Cold Spring Harbor perspectives in biology 4:a005645. 
Salimpoor VN, Benovoy M, Larcher K, Dagher A, Zatorre RJ. 2011. Anatomically distinct dopamine release during anticipation and experience of peak emotion to music. Nat Neurosci 14:257-262.

Salimpoor VN, Benovoy M, Longo G, Cooperstock JR, Zatorre RJ. 2009. The rewarding aspects of music listening are related to degree of emotional arousal. PLoS ONE 4.

Salimpoor VN, van den Bosch I, Kovacevic N, McIntosh AR, Dagher A, Zatorre RJ. 2013. Interactions between the nucleus accumbens and auditory cortices predict music reward value. Science 340:216-219.

Sarkamo T, Tervaniemi M, Laitinen S, Forsblom A, Soinila S, Mikkonen M, Autti T, Silvennoinen HM, Erkkila J, Laine M et al. 2008. Music listening enhances cognitive recovery and mood after middle cerebral artery stroke. Brain 131:866-876.

Scherzer CR, Eklund AC, Morse LJ, Liao Z, Locascio JJ, Fefer D, Schwarzschild MA, Schlossmacher MG, Hauser MA, Vance JM et al. 2007. Molecular markers of early Parkinson's disease based on gene expression in blood. Proceedings of the National Academy of Sciences of the United States of America 104:955-960.

Scherzer CR, Grass JA, Liao Z, Pepivani I, Zheng B, Eklund AC, Ney PA, Ng J, McGoldrick M, Mollenhauer B et al. 2008. GATA transcription factors directly regulate the Parkinson's diseaselinked gene alpha-synuclein. Proceedings of the National Academy of Sciences of the United States of America 105:10907-12.

Schlaug G, Norton A, Overy K, Winner E. 2005. Effects of music training on the child's brain and cognitive development. Annals Of The New York Academy Of Sciences 1060:219-230.

Schubert E, Hargreaves DJ, North AC. 2014. A dynamically minimalist cognitive explanation of musical preference: is familiarity everything? Frontiers in psychology 5:38.

Seashore C, Lewis D, Saetveit J. 1960. Seashore Measures of Musical Talent (Revised).

Sluming V, Brooks J, Howard M, Downes JJ, Roberts N. 2007. Broca's area supports enhanced visuospatial cognition in orchestral musicians. The Journal of neuroscience : the official journal of the Society for Neuroscience 27:3799-3806.

Südhof TC, Rizo J. 2011. Synaptic vesicle exocytosis. Cold Spring Harbor perspectives in biology 3.

Sullivan PF, Fan C, Perou CM. 2006. Evaluating the comparability of gene expression in blood and brain. American journal of medical genetics. Part B, Neuropsychiatric genetics: the official publication of the International Society of Psychiatric Genetics 141B:261-268.

Sutoo D, Akiyama K. 2004. Music improves dopaminergic neurotransmission: demonstration based on the effect of music on blood pressure regulation. Brain Research 1016:255-262. 
Theusch E, Basu A, Gitschier J. 2009. Genome-wide study of families with absolute pitch reveals linkage to 8q24.21 and locus heterogeneity. American journal of human genetics 85:112-9.

Tylee DS, Kawaguchi DM, Glatt SJ. 2013. On the outside, looking in: A review and evaluation of the comparability of blood and brain "-omes." American Journal of Medical Genetics, Part B: Neuropsychiatric Genetics 162:595-603.

Ukkola-Vuoti L, Oikkonen J, Onkamo P, Karma K, Raijas P, Järvelä I. 2011. Association of the arginine vasopressin receptor 1A (AVPR1A) haplotypes with listening to music. Journal of human genetics 56:324-329.

Wada K, Howard JT, McConnell P, Whitney O, Lints T, Rivas M V, Horita H, Patterson MA, White SA, Scharff C et al. 2006. A molecular neuroethological approach for identifying and characterizing a cascade of behaviorally regulated genes. Proceedings of the National Academy of Sciences of the United States of America 103:15212-7.

Wang J, Nicol T, Skoe E, Sams M, Kraus N. 2009. Emotion modulates early auditory response to speech. Journal of cognitive neuroscience 21:2121-2128.

Wong PCM, Skoe E, Russo NM, Dees T, Kraus N. 2007. Musical experience shapes human brainstem encoding of linguistic pitch patterns. Nature neuroscience 10:420-422.

Zhang G, Li C, Li Q, Li B, Larkin DM, Lee C, Storz JF, Antunes A, Greenwold MJ, Meredith RW et al. 2014. Comparative genomics reveals insights into avian genome evolution and adaptation. Science 346:1311-1320.

\section{Figure Legends}

Fig. 1. Differential gene expression in experienced listeners vs 'music-free' controls. Heatplot representations of mean expression values pre- and post-music listening session and control sessions. The red-yellow-green palette represents low-moderate-high expression values. (a) Educated listeners vs controls, (b) Competent listeners vs controls.

Fig. 2. Schematic representation of chromosome 4. The $\alpha$-synuclein gene $(S N C A)$ that was found to be up-regulated after music perception in this study is located in the best linkage region of musical 
505 aptitude as shown by Pulli et al. (Pulli et al., 2008), Park et al. (Park et al., 2012) and Oikkonen et al. 506 (Oikkonen et al., 2014). GATA2, which is located in the best genome-wide association region of 507 musical aptitude) (Oikkonen et al., 2014) and regulates the $S N C A$, is also shown. 
Table $\mathbf{1}$ (on next page)

Phenotype characteristics of the sample set (total $n=48$ )

$n$ represents the number of participants within each category 


\section{PeerJ Reviewing Manuscript}

Table 1. Phenotype characteristics of the sample set (total $n=48$ )

\begin{tabular}{|c|c|}
\hline Phenotype & $\mathbf{n}^{*}$ \\
\hline Edu classes 1-2 & 19 \\
Edu classes 3-4 & 29 \\
Low COMB scores & 12 \\
High COMB scores & 12 \\
Male & 22 \\
Female & 26 \\
\hline
\end{tabular}

* $\mathrm{n}$ represents the number of participants within each category 


\section{Table 2 (on next page)}

Putative biological functions of the differentially expressed genes after listening to music 
Table 2: Putative biological functions of the differentially expressed genes after listening to music

\begin{tabular}{|c|c|c|}
\hline Biological function & Gene & Direction of regulation \\
\hline Dopamine secretion, transport, signaling & SNCA, RTN4, RGS2, SLC6A8 & Up \\
\hline $\begin{array}{c}\text { Synaptic neurotransmission } \\
\text { (Vesicular exocytosis, endocytosis) }\end{array}$ & SNCA, STXBP2, FKBP8, SYNJ1, LYST, SUMO2, HDAC4, DUSP6 & Up \\
\hline Synaptic function & SNCA, NPTN, FKBP8, NRGN, HDAC4 & Up \\
\hline Learning and memory, cognitive performance & SNCA, NRGN, NPTN, FKBP8, RTN4, SLC6A8, NEDD9 & Up \\
\hline Song learning and singing in songbirds & $S N C A, N R G N, R G S 2, M Y C, U B E 2 B$ & Up \\
\hline Auditory cortical activation & HDAC4, LRRFIPI & Up \\
\hline Absolute pitch & FAM49B, HDAC4 & Up \\
\hline Neuroprotection & SNCA, RTN4, FKBP8, SLC6A8, KLF4 & $\mathrm{Up}$ \\
\hline Neurogenesis & KLF4, SMNDC1, S100A12 & Up \\
\hline Neuronal apoptosis & CASP8, GZMH, GZMA, IFI6, PYCARD, TNFRSF10B, HSPE1 & Down \\
\hline ATP synthase coupled proton transport & ATP5J, ATP5L & Down \\
\hline
\end{tabular}




\section{1}

Differential gene expression in experienced listeners vs 'music-free' controls

Heatplot representations of mean expression values pre- and post-music listening session and control sessions. The red-yellow-green palette represents low-moderate-high expression values. (a) Educated listeners vs controls, (b) Competent listeners vs controls.

(a). Down-regulated \& Up-regulated genes in Listeners (edu classes 3-4) VS controls

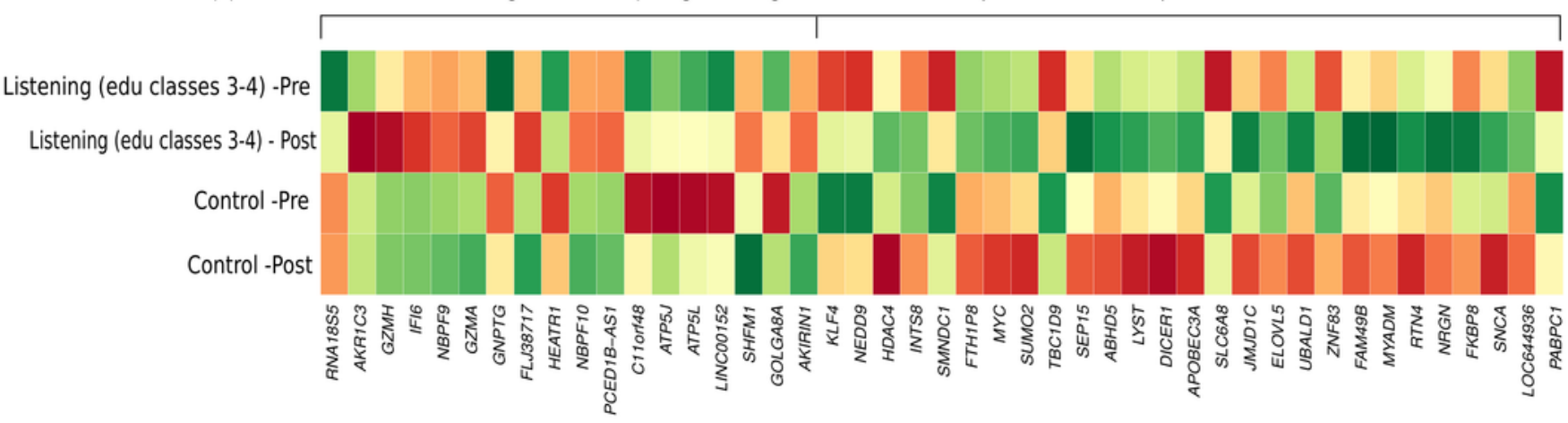

(b). Down-regulated \& Up-regulated genes in Listeners (high COMB scores) VS controls
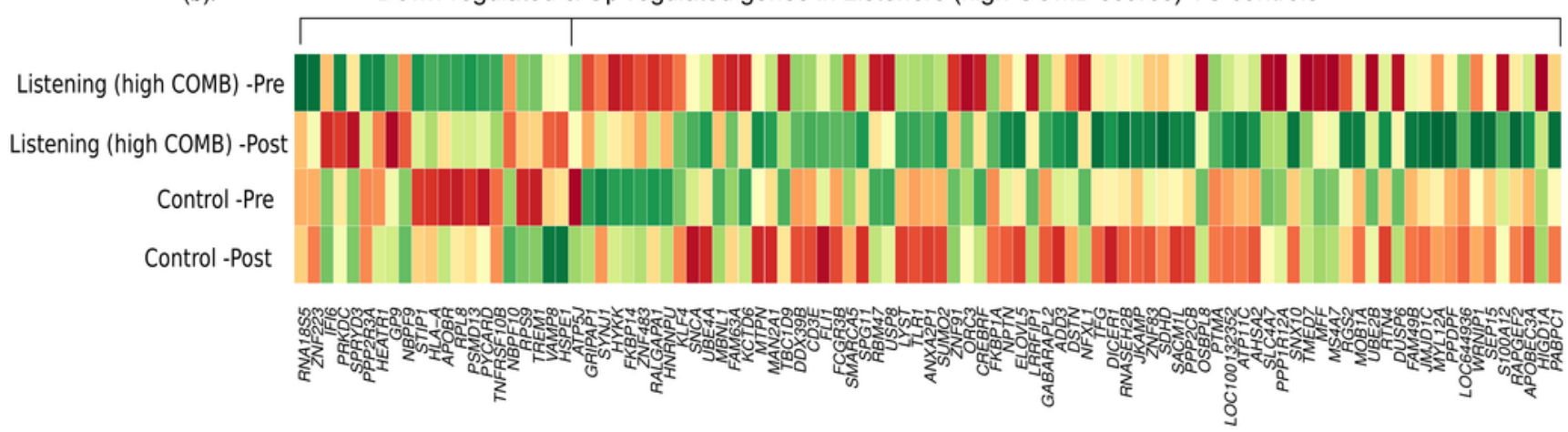


\section{2}

Schematic representation of chromosome 4.

The $\alpha$-synuclein gene (SNCA) that was found to be up-regulated after music perception in this study is located in the best linkage region of musical aptitude as shown by Pulli et al. (Pulli et al., 2008b) , Park et al. (Park et al., 2012) and Oikkonen et al. (Oikkonen et al., 2014b) . GATA2, which is located in the best genome-wide association region of musical aptitude) (Oikkonen et al., 2014b) and regulates the SNCA, is also shown.

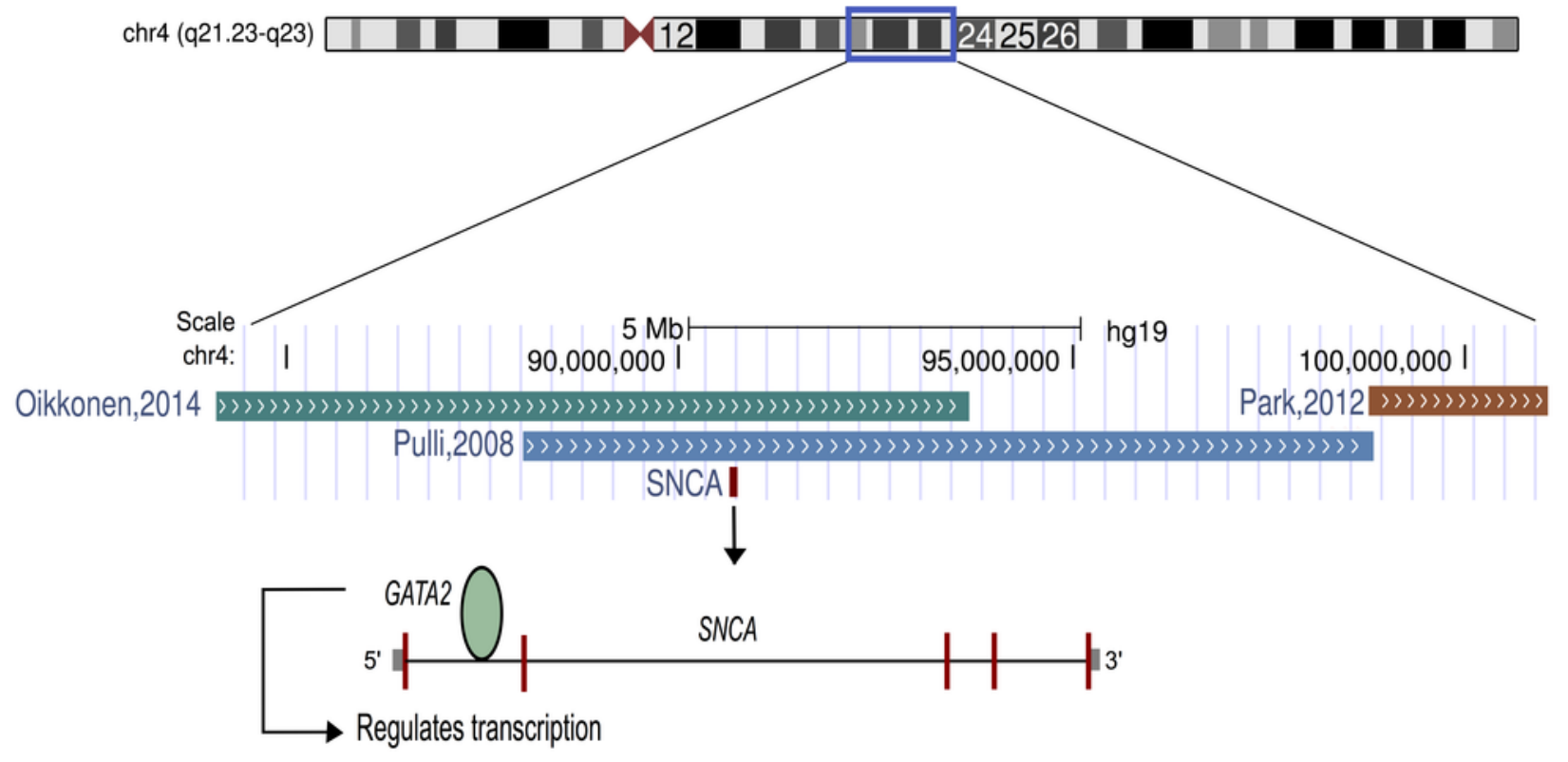

